\title{
The New SuperORRUBA Detector for Transfer Reaction Studies of Exotic Nuclei
}

\author{
D. W. Bardayan ${ }^{a}$, S. $\mathbf{A h n}^{b}$, J. C. Blackmon ${ }^{c}$, A. J. Burkhart ${ }^{d}$, K. Y. Chae ${ }^{a, e}$, \\ J. A. Cizewski ${ }^{* f}$, J. Elson ${ }^{g}$, S. Hardy ${ }^{h}$, R. L. Kozub ${ }^{d}$, L. Linhardt ${ }^{c}$, B. Manning ${ }^{f}$, \\ M. Matoš ${ }^{c}$, S. D. Pain ${ }^{a}$, L. G. Sobotka ${ }^{g}$, M. S. Smith ${ }^{a}$ \\ ${ }^{a}$ Physics Division, Oak Ridge National Laboratory, Oak Ridge, TN 37831, USA \\ ${ }^{b}$ Department of Physics and Astronomy, University of Tennessee, Knoxville, TN 37996, USA \\ ${ }^{c}$ Department of Physics and Astronomy, Louisiana State University, Baton Rouge, LA 70803, USA \\ ${ }^{d}$ Physics Department, Tennessee Technological University, Cookeville, TN 38505, USA \\ ${ }^{e}$ Department of Physics, Sungkyunkwan University, Suwon 440-746, Korea \\ ${ }^{f}$ Department of Physics and Astronomy, Rutgers University, New Brunswick, NJ 08903, USA \\ ${ }^{g}$ Department of Chemistry, Washington University, Saint Louis, MO 63130, USA \\ ${ }^{h}$ Department of Physics, University of Surrey, Guildford, Surrey, GU2 7XH, UK \\ Email: bardayandw@ornl.gov
}

\begin{abstract}
The astrophysical rapid neutron-capture process (r-process) is recognized as the scenario responsible for the synthesis of approximately half of the nuclear species more massive than Fe. Unfortunately, almost nothing is known about the structure of the majority of the extremely neutron-rich nuclei involved in the r-process reaction flow. At exotic beam facilities such as the Holifield Radioactive Ion Beam Facility (HRIBF), measurements with accelerated beams of fission fragments have provided some of the first spectroscopic information on many r-process nuclei. In particular, the study of transfer reactions in inverse kinematics has yielded important spectroscopic information. While these measurements are experimentally challenging, they can be facilitated by surrounding the target by a large array of segmented silicon detectors. The SuperORRUBA (Oak Ridge Rutgers University Barrel Array) detector has been constructed at Oak Ridge National Laboratory (ORNL) for this purpose. The detector is now in routine use and has made possible several new measurements at the HRIBF.
\end{abstract}

XII International Symposium on Nuclei in the Cosmos,

August 5-12, 2012

Cairns, Australia

${ }^{*}$ Speaker. 


\section{Introduction}

The development of exotic beam facilities has created opportunities for some of the first studies of many short-lived nuclei of interest for nuclear astrophysics [1]. The r-process involves thousands of neutron-rich nuclei about which very little is known. As it is clear that all of these nuclei cannot be studied experimentally, it is critical to benchmark nuclear structure models upon which calculations of r-process nucleosythesis must rely. In addition, individual neutron-capture rates can significantly impact late-time processing [2,3], and thus better nuclear structure information is needed on these nuclei to constrain the astrophysical reaction rates.

A powerful technique is to study the single-neutron transfer reaction $(d, p)$ induced by bombarding exotic beams on light deuterium-containing targets (inverse kinematics) $[4,5]$. The ejected protons from such reactions carry the nuclear spectroscopic information of interest. Therefore it is critical to detect, identify, and accurately measure their energies and angles of emission. The difficulties with such measurements are illustrated in Fig. 1. Because of the extreme inverse nature of the reaction, the proton energy rapidly changes as a function of laboratory angle, necessitating the need for accurate emission angle measurements. Large solid angle coverage is also required because of the relatively low intensity of exotic beams.

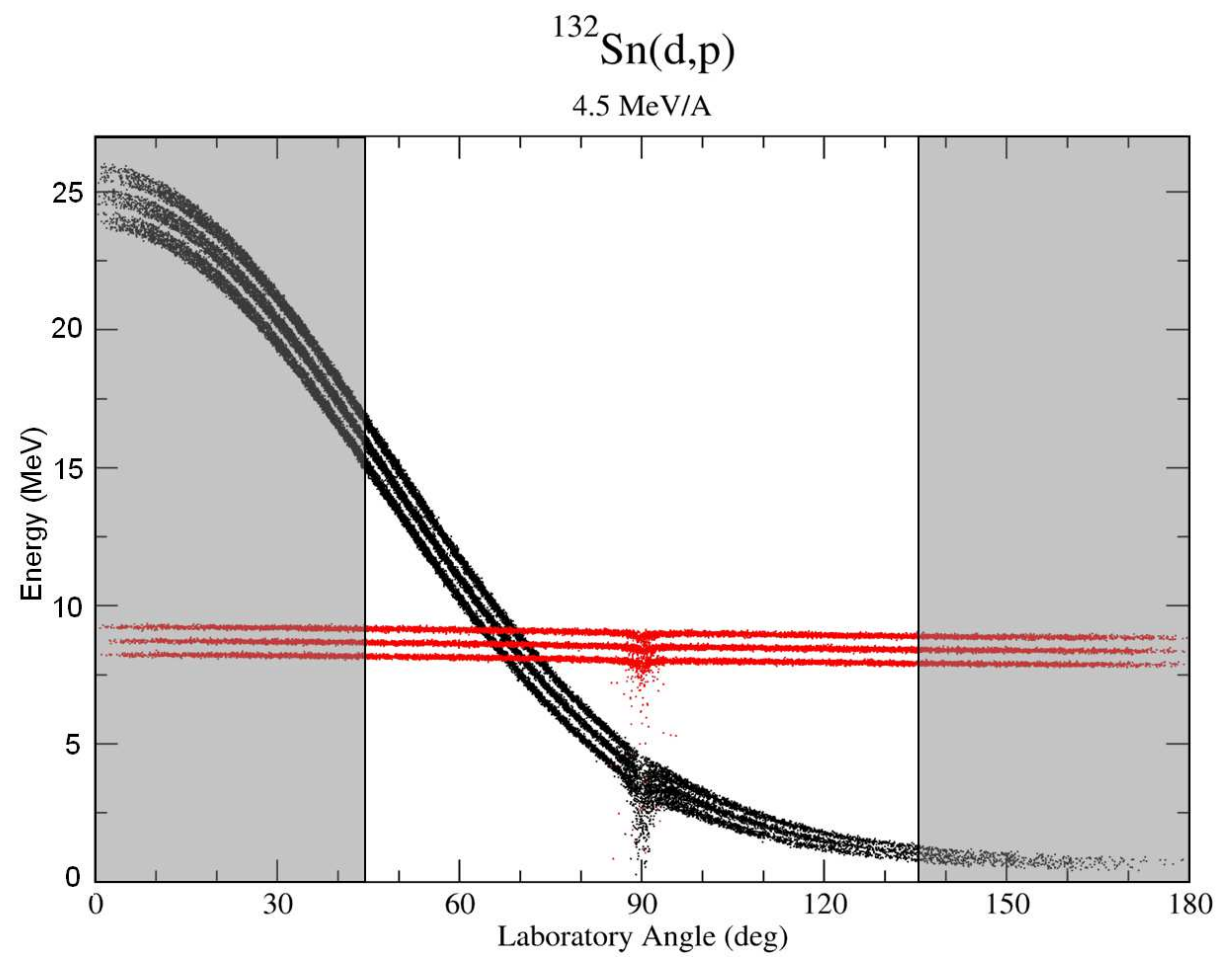

Figure 1: Expected energies of protons from the ${ }^{2} \mathrm{H}\left({ }^{132} \mathrm{Sn}, p\right){ }^{133} \mathrm{Sn}$ reaction at $4.5 \mathrm{MeV} / \mathrm{A}$ calculated with a Monte Carlo code and accounting for energy loss and straggling in the target, detector energy resolution, and assuming 1 degree angular bin sizes for the population of hypothetical states at $0,0.5$, and $1 \mathrm{MeV}$ excitation energies. Simulations are shown for both normal (horizontal) and inverse (vertical) kinematics. The area of the plot highlighted in white shows the approximate polar angular range in which detection of protons is practical in inverse kinematics. 


\section{The SuperORRUBA Detector}

To meet these demanding requirements, the SuperORRUBA (Oak Ridge Rutgers University Barrel Array) detector has been developed at Oak Ridge National Laboratory (ORNL). The detector was built upon the original design of the ORRUBA [6] detector but with the greater sensitivity and resolution made possible with double-sided silicon strip technology [7]. An early implementation of the ORRUBA detector along with the Silicon Detector Array (SIDAR) [8] was used for inverse-kinematics studies of the ${ }^{132} \mathrm{Sn}(d, p){ }^{133} \mathrm{Sn}[9],{ }^{130} \mathrm{Sn}(d, p){ }^{131} \mathrm{Sn}[10]$, and ${ }^{134} \mathrm{Te}(d, p){ }^{135} \mathrm{Te}$ [11] reactions. The ORRUBA array was first fully implemented for a study of the ${ }^{10} \mathrm{Be}(d, p){ }^{11} \mathrm{Be}$ reaction [12].

Elements of the new SuperORRUBA detector cover the same geometrical area as the ORRUBA detector elements, $7.5 \mathrm{~cm} \times 4 \mathrm{~cm}$, but the front sides were divided into $641.2 \mathrm{~mm} \times 4$ $\mathrm{cm}$ strips, and the back sides were segmented into $47.5 \mathrm{~cm} \times 1 \mathrm{~cm}$ strips. The detectors were manufactured by Micron Semiconductor [13] (Micron style BB15). As a result of the significantly increased number of detector strips that needed to be connected to the detector header, the detector PCB board width had to be increased to $59 \mathrm{~mm}$ while the length was maintained at $103 \mathrm{~mm}$. Similar to the ORRUBA detectors, the individual elements were assembled into two dodecagon rings (Fig. 2), one forward of $90^{\circ}$ in the laboratory and one backward. The radius of the forward (backward) angle ring was $11.2(12.5) \mathrm{cm}$, respectively. A small space between the two was left such that the forward and backward rings could be overlapped with no loss in polar angle coverage between them. When fully instrumented, the SuperORRUBA detector has 70\% azimuthal coverage at forward angles and $60 \%$ azimuthal coverage at backward angles.

Signal processing was performed with Application-Specific Integrated Circuits (ASICs) electronics designed at Washington University in St. Louis and Southern Illinois University Edwardsville [14]. Preamplifier signals were routed to ASIC chipboards capable of shaping, triggering, and timing 32 independent channels. Up to 16 chipboards can populate an ASICs motherboard; 5 motherboards were implemented with the data acquisition system allowing for maximum channel count of 2560. Data rates on the order of $1 \mathrm{kHz}$ were acquired with $<30 \%$ deadtime.

\section{First results}

Initial tests with a calibrated triple $\alpha\left({ }^{239} \mathrm{Pu},{ }^{241} \mathrm{Am},{ }^{244} \mathrm{Cm}\right)$ source revealed an intrinsic detector resolution of $25 \mathrm{keV}$. Subsequent in-beam tests demonstrated that center of mass resolutions of $200 \mathrm{keV}$ could be obtained with heavy-ion beams on light-ion targets. Figure 3 shows the proton energy spectrum observed as a function of laboratory angle for a $630-\mathrm{MeV}{ }^{124} \mathrm{Sn}$ beam on a $139 \mu \mathrm{g} / \mathrm{cm}^{2} \mathrm{CD}_{2}$ target. Several bands produced by the $(d, p)$ reaction are evident. The resolution obtained is consistent with simulations taking into account target thickness, angular bin size, and intrinsic detector resolution.

After initial tests with stable beams, several experiments were performed at the Holifield Radioactive Ion Beam Facility (HRIBF) with exotic beams. The ${ }^{26} \mathrm{Al}(d, p)^{27} \mathrm{Al}$ [15] reaction was studied to better understand the astrophysical ${ }^{26} \mathrm{Al}(p, \gamma){ }^{27} \mathrm{Si}$ reaction rate. $\mathrm{A}^{80} \mathrm{Ge}(d, p){ }^{81} \mathrm{Ge}$ study of the $\mathrm{N}=49$ nucleus, ${ }^{81} \mathrm{Ge}$, has been performed to confirm spins and parities of low-lying levels [16]. The ${ }^{126} \operatorname{Sn}(d, p){ }^{127} \mathrm{Sn}$ and ${ }^{128} \operatorname{Sn}(d, p){ }^{129} \mathrm{Sn}[17,18]$ reactions have been measured completing 

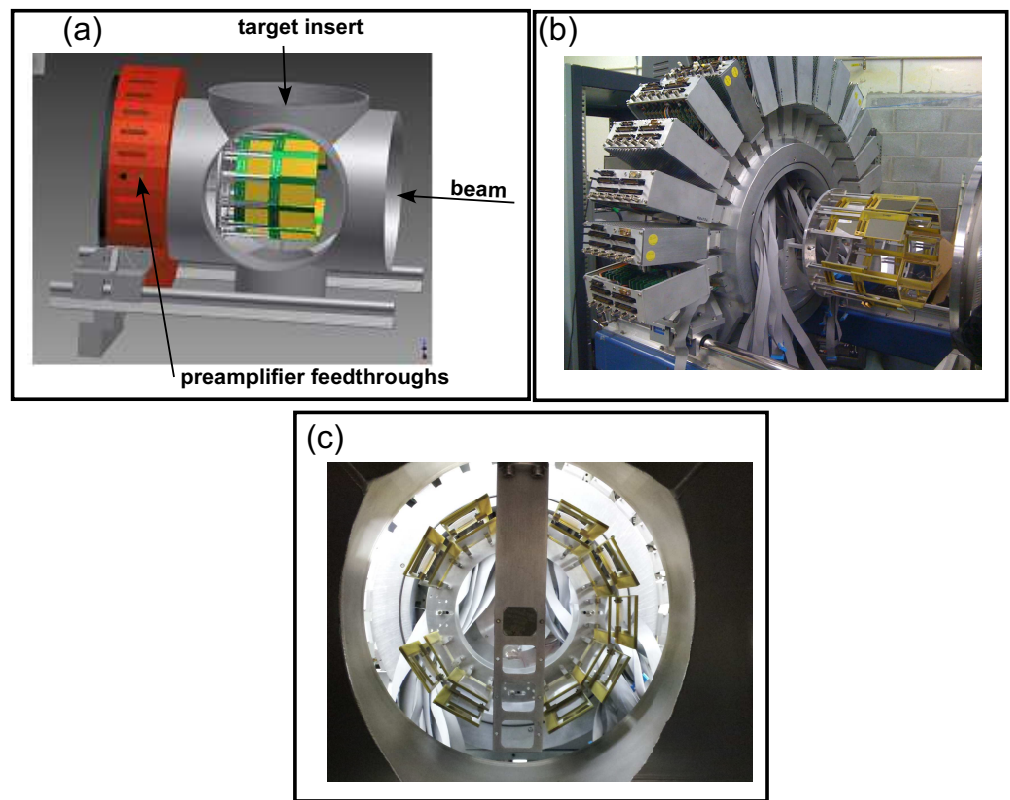

Figure 2: (a) Design drawing of the SuperORRUBA assembly with the preamplifier ring and detector inserted into the target chamber. (b) Photo of the assembly pulled out of the target chamber and with preamplifier units mounted. (c) Beam view photo to illustrate how the detector surrounds the target-beam interaction point.

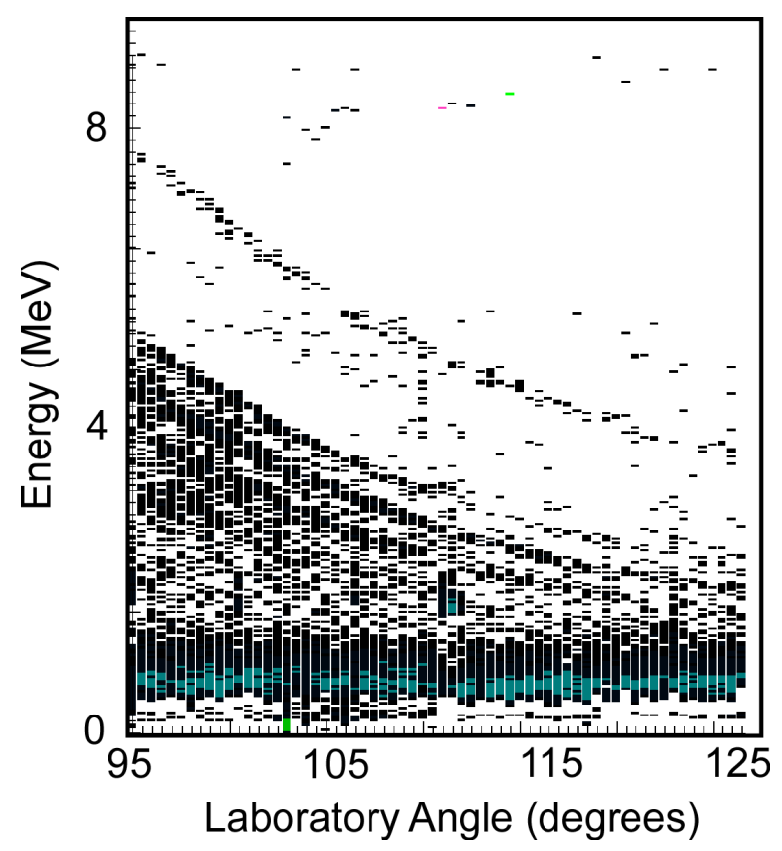

Figure 3: Energy of protons from a bombardment of a $139 \mu \mathrm{g} / \mathrm{cm}^{2} \mathrm{CD}_{2}$ target with a $630-\mathrm{MeV}^{124} \mathrm{Sn}$ beam as detected by the new SuperORRUBA detector and in coincidence with forward-going recoils. Several bands are evident from the population of excited ${ }^{125} \mathrm{Sn}$ states from the $(d, p)$ reaction. 


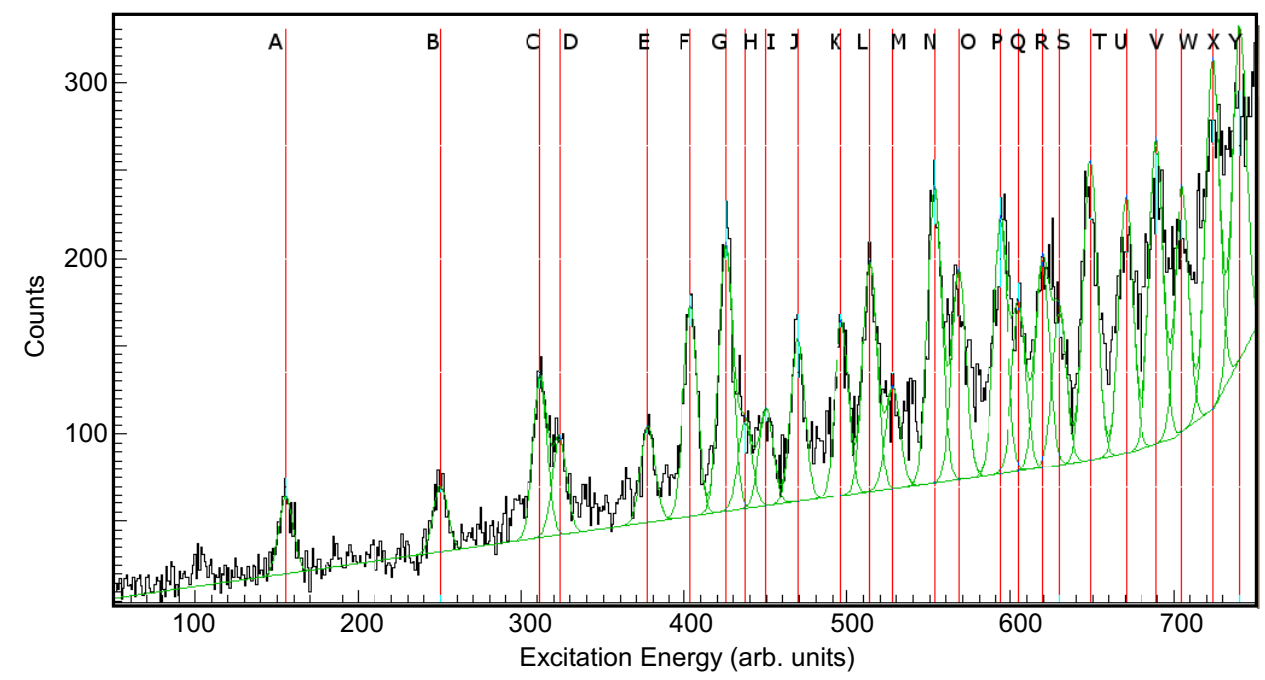

Figure 4: The excitation energy of ${ }^{27} \mathrm{Al}$ levels observed in an ${ }^{26} \mathrm{Al}(d, p){ }^{27} \mathrm{Al}$ measurement. States labeled A-Y correspond to excited ${ }^{27} \mathrm{Al}$ levels in the excitation energy range 3-11 MeV, respectively. The final calibration is in progress. The observed $Q$-value resolution was approximately $170 \mathrm{keV}$.

our studies of $(d, p)$ reactions on even-mass Sn isotopes from ${ }^{124} \mathrm{Sn}$ to ${ }^{132} \mathrm{Sn}$. Finally the first neutron removal reaction has been measured on doubly-magic ${ }^{132} \mathrm{Sn}$ via a study of the ${ }^{132} \mathrm{Sn}(d, t){ }^{131} \mathrm{Sn}$ reaction at the HRIBF. Data from the ${ }^{26} \mathrm{Al}(d, p)^{27} \mathrm{Al}$ measurement at $\theta_{c . m .}=33^{\circ}$ are shown in Fig. 4 and were obtained from the bombardment of a $177-\mathrm{MeV}^{26} \mathrm{Al}$ beam on a $100 \mu \mathrm{g} / \mathrm{cm}^{2} \mathrm{CD}_{2}$ target. The obtained energy resolution $(170 \mathrm{keV})$ was somewhat better in this experiment owing to the reduced energy loss in the target.

\section{Conclusions}

A new detector has been built to detect reaction products from transfer reactions induced by exotic beams bombarding light targets. These studies benefit from the detection of charged particles near $90^{\circ}$ in the laboratory with good energy and angular resolution and large solid-angle coverage. The array has been constructed and commissioned at the HRIBF achieving $25 \mathrm{keV}$ intrinsic resolution. In a beam study of the ${ }^{124} \mathrm{Sn}(d, p){ }^{125} \mathrm{Sn}$ and ${ }^{26} \mathrm{Al}(d, p)^{27} \mathrm{Al}$ reactions, 200-keV and $170-\mathrm{keV}$ resolutions were obtained, respectively, primarily due to the target energy loss and harsh kinematic broadening near $90^{\circ}$.

In the future, the detector will be used with the Jet Experiments for Nuclear Structure and Astrophysics (JENSA) gas-jet target, which is currently under construction at ORNL [19]. The gasjet target in combination with SuperORRUBA is planned for studies of $\left({ }^{3} \mathrm{He}, d\right)$ and other transfer reactions on radioactive beams at the ReA3 facility at Michigan State University [20].

This work was supported in part by the U.S. Department of Energy (DOE) Office of Nuclear Physics under Contract Nos. DE-AC05-00OR22725 (ORNL), DE-FG02-96ER40955 (TTU), DEFG02-96ER40983 and DE-SC0001174 (UT), DE-FG02-87ER-40316 (WU), the National Nuclear Security Administration under the Stewardship Science Academic Alliance program through DOE 
Cooperative Agreement No. DE-FG52-08NA28552 (Rutgers), and by the National Science Foundation (Rutgers).

\section{References}

[1] M. S. Smith and K. E. Rehm, Annu. Rev. Nucl. Part. Sci. 51 (2001) 91.

[2] J. Beun, J. C. Blackmon, W. R. Hix, G. C. McLaughlin, M. S. Smith, R. Surman, J. Phys. G 36 (2009) 025201 .

[3] R. Surman, J. Beun, G. C. McLaughlin, W. R. Hix, Phys. Rev. C 79 (2009) 045809.

[4] W. N. Catford, Nucl. Phys. A 701 (2002) 1c.

[5] K. E. Rehm et al., Phys. Rev. Lett. 80 (1998) 676.

[6] S. D. Pain et al., Nucl. Instr. and Meth. B 261 (2007) 1122.

[7] D. W. Bardayan et al., Nucl. Instr. and Meth. A (submitted).

[8] D. W. Bardayan et al., Phys. Rev. C 63 (2001) 065802.

[9] K. L. Jones et al., Nature 465 (2010) 454; K. L. Jones et al., Phys. Rev. C 84 (2011) 034601.

[10] R. L. Kozub et al., Phys. Rev. Lett. (in press).

[11] S. D. Pain et al., PoS (NIC X) (2008) 142.

[12] K. T. Schmitt et al., Phys. Rev. Lett. 108 (2012) 192701.

[13] Micron Semiconductor, http://www.micronsemiconductor.co.uk/.

[14] G. L. Engel, M. Sadasivam, M. Nethi, J. M. Elson, L. G. Sobotka, R. J. Charity, Nucl. Instr. and Meth. A 573 (2007) 418.

[15] S. Hardy, M. Phys. thesis, U. Surrey (2012).

[16] S. H. Ahn et al., Bull. Amer. Phys. Soc. 56, No. 4 (2011) 193.

[17] B. Manning et al., Bull. Amer. Phys. Soc. 56, No. 12 (2011).

[18] J. A. Cizewski et al. in these proceedings.

[19] K. A. Chipps et al., Bull. Amer. Phys. Soc. 56, No. 12 (2011) 68.

[20] S. Schwarz et al., Hyperfine Interact. 199 (2011) 261.33. 